\title{
Outcome of medial sphenoidal wing meningioma surgery
}




\title{
Outcome of medial sphenoidal wing meningioma surgery
}

\author{
Ashraf El Badry', Azza Abdelazeez ${ }^{2}$ \\ ${ }^{1}$ Neurosurgery Department; ${ }^{2}$ Pathology Department \\ Faculty of Medicine, Mansoura University Hospital, EGYPT
}

\begin{abstract}
Background: Sphenoid wing meningiomas among difficult surgery tumours due to involvement of important structures like optic nerve, cavernous sinus or carotid artery. Objective: to evaluate outcome and prognostic factors in patients with medial sphenoid wing meningiomas after microsurgery in a series of 47 patients with medial sphenoid wing meningioma. Method: This paper includes analysis of 47 patients \& their data who underwent surgical management for meningiomas involving the medial sphenoid wing in the period between Jan 2008 and February 2016 at Mansoura university hospital. Results: The most of patients were suffering from visual impairment (59.6\%), followed by headaches (38.2\%). Total tumor resection were achieved in 23 cases, incomplete in 19 cases and partial resection in 5 cases. Visual acuity improved or stable in $57.4 \%$ of the patients. Preoperative neurological deficit remains unchanged postoperative in 35 cases while improved in 4 cases. 8 cases developed permanent $3 \mathrm{rd}$ Nerve palsy. Tumor recurrence was in 7 patients. Conclusion: There are many factors influence the prognosis of inner sphenoid wing meningioma including the extent of surgical resection, cavernous sinus invasion, present or absent of peritumoral edema, vascularity of the tumor, size of the tumor, presence or absence of arachnoid plane between the tumor and surrounding neurovascular structures and preoperative neurological condition
\end{abstract}

Key words: sphenoid, meningioma, inner wing, surgery

\section{Introduction}

The intracranial meningioma presumed to represent about 14 to $18 \%$ of all intracranial neoplasms. The sphenoid ridge meningioma account approximately $20 \%$ of all supratentorial meningioma, on the other hand the half of it occurred in the medial sphenoid ridge [5]. It was termed for the first time by Cushing and Eisenhardt, differentiating between two forms: globoid tumors that poses nodular nature \& meningioma en plaque variety, which had a flat shape (sheet) and spread alongside the sphenoid wing. The globoid tumours additional divided into three categories (Figure 1): medial group, middle group \& lateral group. The medial group 
(Figure 2) appeared in the segment adjacent to the anterior clinoid process. This particular meningiomas had an inherited surgical difficulties, morbidities \& mortalities due to its intimate relations to optic nerve, cavernous sinus, cranial nerves that enter superior orbital fissure and arteries of anterior circulation. [14] On the other hand, the extent of tumours resection still one of major factor influencing the recurrence rate which may alter the long term outcome of those patients making operative procedures a challenge to skull base neurosurgeons all over the world in spite of advancement in microscopes, microsurgical tools and neurosurgery equipment. On the light of this concepts, many neurosurgery preferred to remove these tumours partially or grossly subtotally then refer the patients to radiosurgery. [13,17]

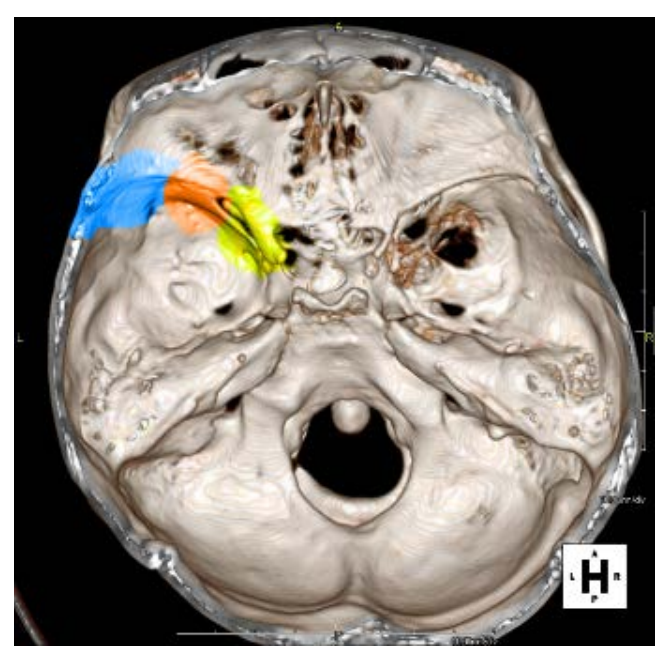

Figure 1 - Diagram show the three tires of Sphenoid wing meningioma according to the location: medial group (yellow), middle group (orange), lateral group (blue)

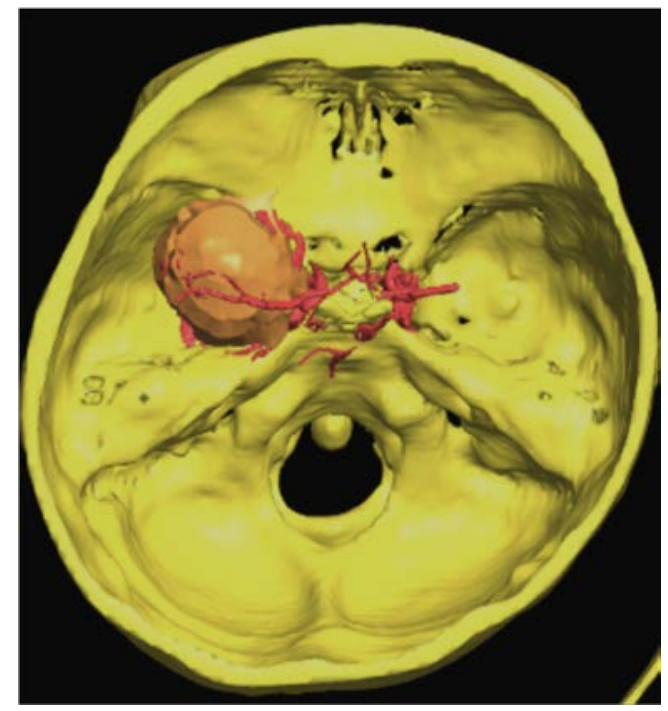

Figure 2 - diagram reveal the site of dural base to the medial sphenoid wing meningioma

The decision making became more difficult to get balance between surgical morbidity \& mortality and recurrent rate by adopting a good microsurgical technique maintaining cranial nerves \& skull base vessels integrity.

Our goals in this paper: to evaluate outcome and prognostic factors in patients with medial sphenoid wing meningiomas after microsurgery \& application of current neurosurgical equipment in a series of 47 patients with medial sphenoid wing meningioma retrospectively.

\section{Patients \& method}

This paper includes analysis of 47 patients \& their data who underwent surgical management for meningiomas involving the medial sphenoid wing (the location was defined by preoperative imaging \& intraoperative report while the pathology was 
confirmed by histological assessment) in the period between Jan 2008 and February 2016 at Mansoura university hospital. All cases that involved middle \& lateral two thirds of sphenoid wing were excluded. Also, we excluded all meningiomas enplaque in this paper as it is not limited to medial sphenoid wing beside it is specific entity that different from our interest of globoid medial sphenoid wing meningioma (did not obey the three tires classification) and the surgical procedures for these tumors are entirely different. The cases who lost in follow up less than 3 months or incomplete data were also eliminated from our study. The follow-up period varied from 3-60 months (mean 14.7 months).

\section{Patients' demographic data}

35 women and 12 men, Mean age of 51.3 years (vary between 32 to 68 years). The Clinical pictures of these cases (Table 1) as follow: visual impairment 59.6\%, headaches $38.2 \%$, 3rd Nerve palsy $8.5 \%$, limb weakness in three cases, proptosis appeared in two cases and epilepsy manifested in two cases.

\section{TABLE 1}

\section{Patients' demographic data}

\begin{tabular}{|c|c|c|}
\hline Clinical presentation & Number of cases & $\%$ \\
\hline visual impairment & 28 & $59.6 \%$ \\
\hline headaches & 18 & $38.2 \%$ \\
\hline 3rd Nerve palsy & 4 & $8.5 \%$ \\
\hline limb weakness & 3 & $6.4 \%$ \\
\hline proptosis & 2 & $4.26 \%$ \\
\hline epilepsy & 2 & $4.26 \%$ \\
\hline
\end{tabular}

All patients who were involved in our research had a complete data including:

$>$ Complete clinical neurological \& ophthalmological (visual acuity) examination

$>$ Computed tomography "thin cuts "on skull base to visualize bony skull base anatomical land marks

$>$ MRI imaging with its variety: T1WE (pre \& post contrast) to delineate the tumor and visualize its relations to the proximate neurovascular structures \& optic nerves while T2WE determine the integrity of arachnoid plane \& gave a rough idea about tumor consistency i.e. hypointense may be associated with tumor firmness

> CTA, MRA or traditional Angiography was done to show tumor feeders and to determine the extent of encasement of the internal carotid artery and its branches by the tumor

$>$ Surgical archives, discharge sheets, histological reports, follow-up records, and post-operative imaging findings.

Patients' radiological data: The mean tumor diameter was $5.7 \mathrm{~cm}$ (Centimeter) and varied between $3.2 \mathrm{~cm} \& 6.1 \mathrm{~cm}$. The peritumoral edema was present in 19 cases (40.4\%) while the med line shift was seen in 15 cases (32\%). the calcifications appeared in 6 cases (12.8\%). 24 cases (51.06\%) showed ipsilateral Invasion of the cavernous sinus. the involvement of the optic nerve canal was seen in 5 cases $(10.6 \%)$

The extent of tumor resection was determined according to the Simpson grading scale (Grade I: total removal with its dural 
attachment, Grade II: total removal with coagulation of dural base, Grade III: subtotal excision...etc.). The post-operative Functional outcomes were established according to the KPS scale. The patients who had a high rank KPS (80-100) who depend on himself to do normal daily activities (4). KPS was used to evaluate all patients (pre \& post) operatively and in follow-up assessments. [5]

\section{Surgical techniques:}

The main objectives of the surgical procedures in our patients were decompression of the optic nerve and complete resection of the tumor intradurally with or without elimination of the intracavernous tumor portion.

In our series, Pterional approach was applied in 36 cases while Orbito-Zygomatic approach was performed in 11 patients

(1) Optimal patient positioning: the patient was placed in supine position. The head was rotated to contralateral side of the procedure with angle ranged from $30 \mathrm{o}$ to $60 \mathrm{o}$ with elevated head of the bed about $15^{\circ}$.

(2) Incision: the shape of the scalp incision varied: question mark flap behind the hair line or bi-coronal skin flap but both must reach by sub fascial dissection to the orbital rim anteriorly and zygomatic arch inferiorly

(3) Bone flap: traditional pterional bone flap, or extended Orbito-Zygomatic bone flap with added orbital rim \& zygomatic arch.

(4) Sphenoid ridge drilling: we removed the outer and middle portion of sphenoid wing or even to anterior clinoid process if necessary, extradurally using high speed drill. To get space and minimize brain retraction

(5) Tumor devascularization: through coagulation of the basal dura after elevation of it under microscopic visualization to decrease the external carotid artery tumor feeders.

(6) Opening the sylvian fissure meticulously

(7) Early localization, revealing and decompression of the ON (Optic Nerve) and ICA (Internal Carotid Artery)

(8) Tracking the ON and ICA to the tumor and explore it

(9) Internal tumor debulking by piece meal or using CAUSA (Cavitron Ultrasonic Surgical Aspirator)

(10) Meticulous extracapsular devascularization and dissection

(11) Preservation of the adherent/adjacent neurovasculature. We depend on maintain the patency of nutrient feeders to the $\mathrm{ON}$ after its decompression for achieving a functioning ON post-surgery.

(12) We de-roofed the Optic Canal and /or Superior Orbital Fissure if the tumor extend in it to relieve the compression on these nerves

(13) If arachnoid plain between (the ICA \& its branches) and the tumour was intact thus assisting in dissection of these vessels easily Peel off the tumour

(14) Meticulous managing cavernous sinus CS involved part of the tumor by removing it if partial CS involvement or leaving this part if excessive invasion of CS by the tumor to avoid high possibility of cranial nerves destruction.

(15) Removal of the involved bone and tumor dural base if possible

(16) Dural reconstruction by fascia late or artificial dura and closure in water tight fashion.

(17) Closure of the bone flap

(18) Anatomical closure 


\section{Case illustration}

49 years old lady had a complaint of sever persistent frontal headache not responding to medical treatment followed by visual deterioration on right eye. Visual premetry revealed enlargement of the left blind spot and left upper inner quadrantanopia. MRI images revealed left medial sphenoid wing meningioma iso to hypo intense with homogenous enhancement that encasing Rt. ICA and compressing the Rt. ON (Figure 3). Traditional Angiography (Figure 4) showed the feeders of the tumor that include: frontal branch of MMA (Middle Meningeal Artery) from Rt. ECA \& meningo-hypophyseal a. which is branch of tentorial a. from ICA. It did not show any constriction in Rt. ICA. We adopt pterional Approach (Figure 5). The surgical technique was as described earlier. Through the steps with microscopic tools we removed the tumour totally with peeling off the tumour from Rt. ICA \& Rt. ON and preserving the nutrient vessels to all the Optic Apparatus as it is crucial steps to maintain vision. Post operatively the vision improved by visual premetry assessment. Post-operative CT Brain axial cuts reveal complete removal of the tumour.

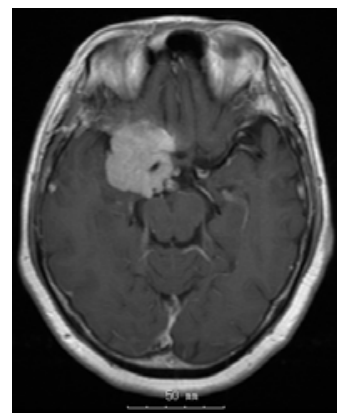

A

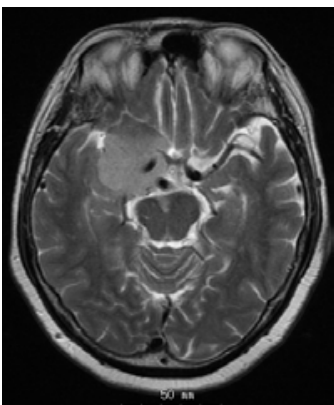

B

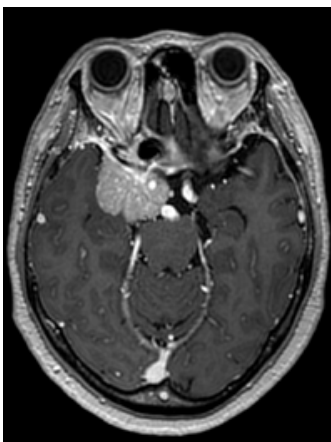

E

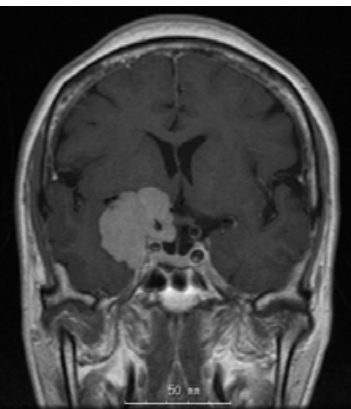

C

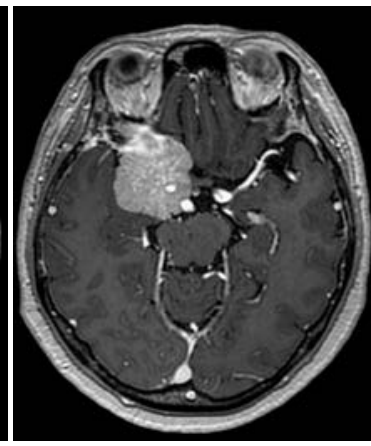

$\mathbf{F}$

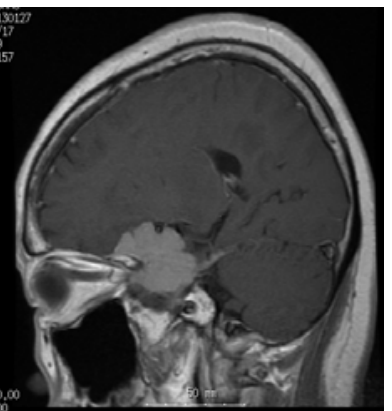

D

Figure 3 - pre-operative Brain MRI images of Rt. Medial Sphenoid Wing meningioma that compressing Rt, ON, Encasing Rt, ICA and extending to the sellar region. A. MRI Brain T1WE axial cut. B. MRI Brain T2WE axial cut that showed iso to hypo intensity of the tumour that indicate some degree of tumour softening. C \& D. MRI Brain

T1WE coronal cuts. E \& F MRI Brain T1WE post contrast axial cuts display homogenous enhancement that indicate high vascularity 


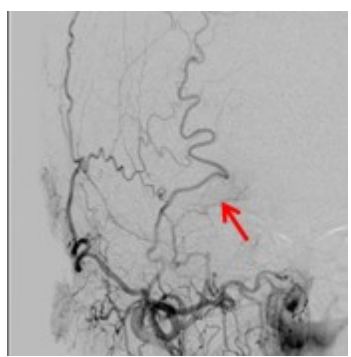

A

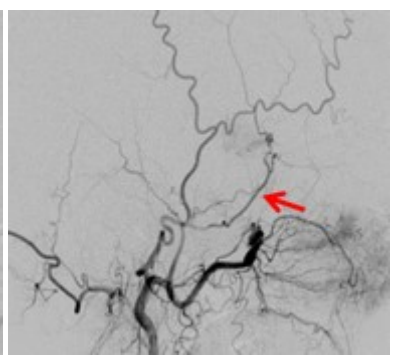

B

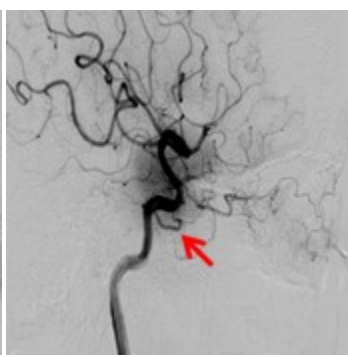

C

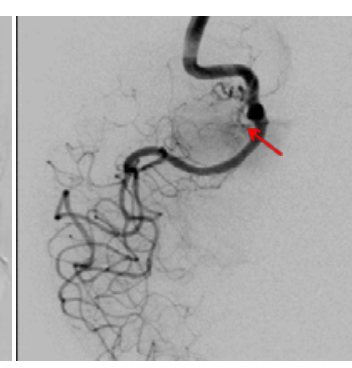

D

Figure 4 - Rt. ECA \& Rt. ICA Angiography images A-D: (A-B) From Rt. ECA, the frontal of MMA feeding the tumor (C-D) From Rt. ICA, meningo-hypophyseal A. branch of tentorial A. give feeder to the tumour

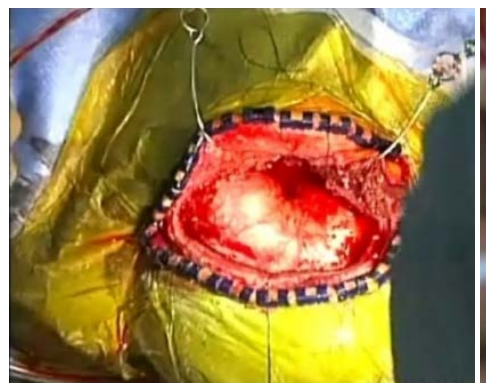

A

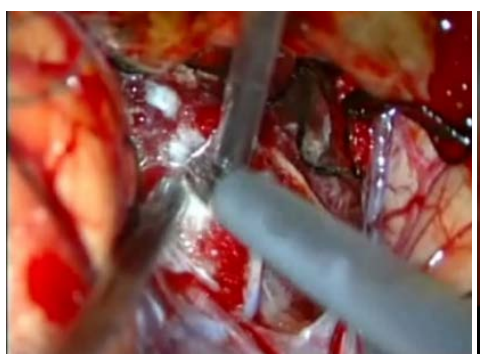

D

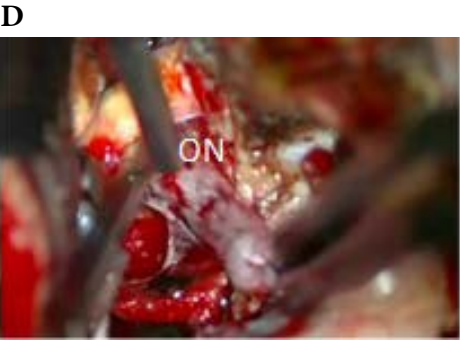

G

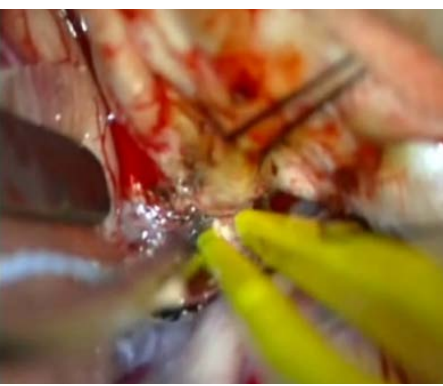

B

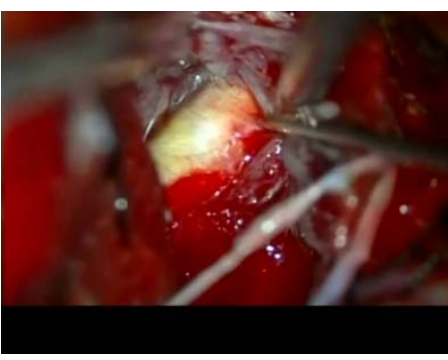

E

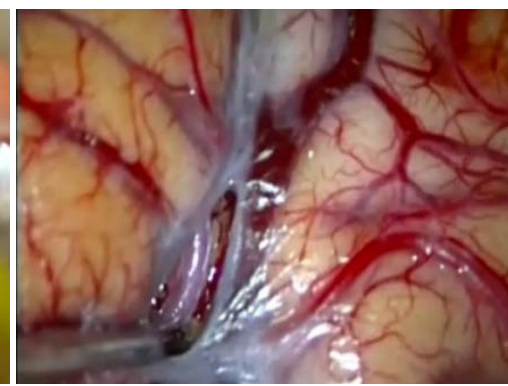

C

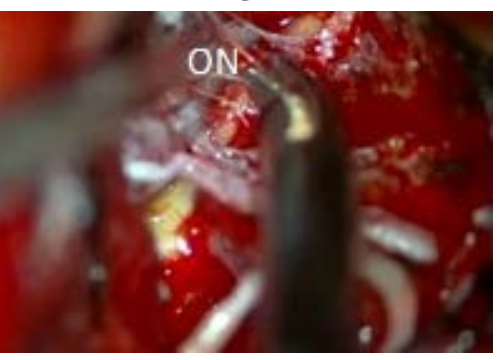

F

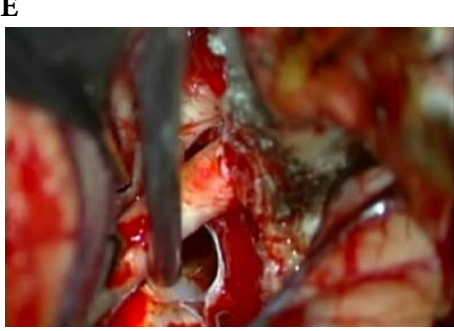

$\mathbf{H}$

Figure 5 - A intraoperative picture reveal drilled sphenoid wing bone till ant. Clinoid and appearance of the whole dural flap. B microscopic picture showed coagulation of the tumour feeders in the tumour dural base by using the bipolar cautery. C. opening the sylvian fissure under the microscope. D. internal debulking of the tumour using CUSA under microscope. E .peeling the tumour off the Optic nerve. F using the micro-scissor to complete the removal of the tumour that encasing the Optic nerve. G. Removing the tumour underneath the Optic nerve \& in the sellar area. H. microscopic picture showed complete tumour excision 


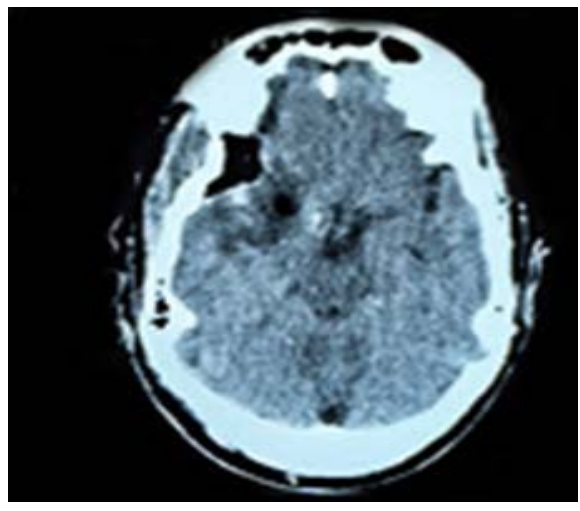

Figure 6 - Post-operative CT Brain axial cut

display complete removal of the tumour

2nd case pre \& post-operative presented in (Figures 7, 8, 9) with complete excision of the tumor:
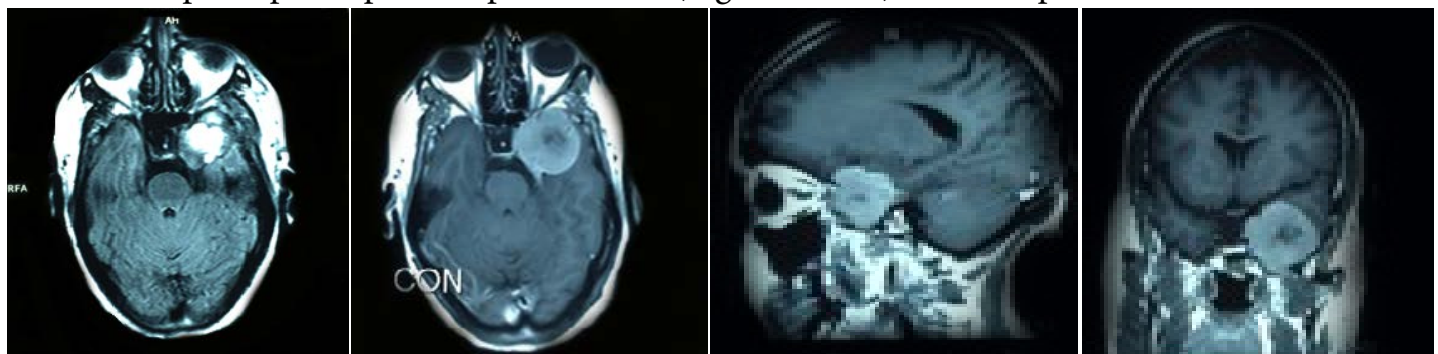

Figure 7 - Second case of medial Sphenoid wing meningioma preoperative MRI images T1WE axial, sagittal and coronal cuts, pre \& post contrast

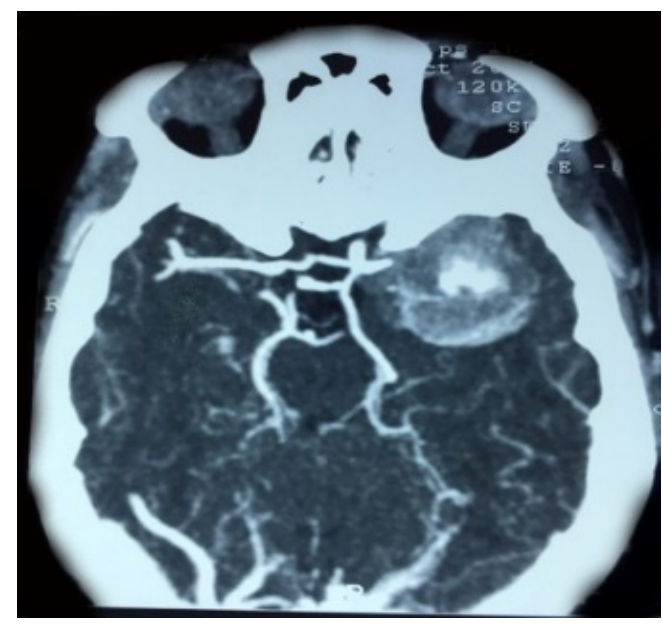

Figure 8 - Pre-operative CT Brain Angiography of 2nd case show encasement of ICA \& MCA (Middle Cerebral Artery) by the tumour and show some tumour calcification

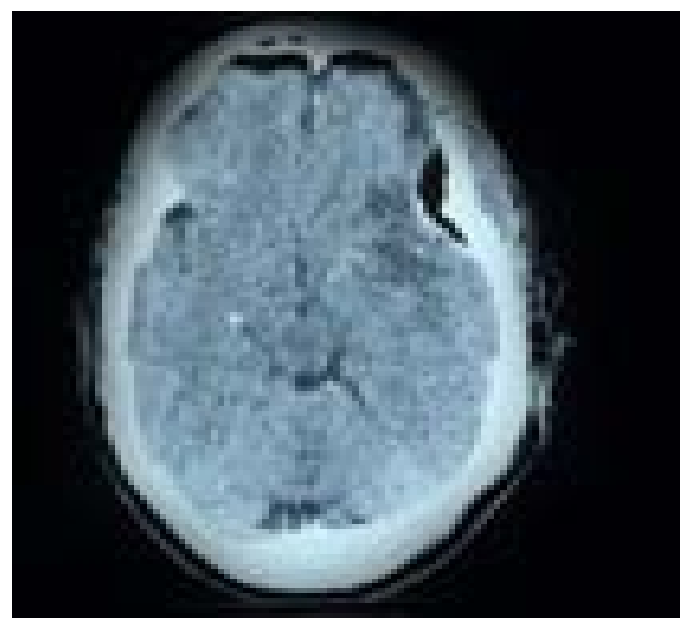

Figure 9 - Post-operative 2nd case CT Brain axial cut display complete tumour resection 
The last presentation case pre-operative MRI Brain axial \& coronal images in (Figure 10), postoperative CT Brain axial cuts (Figure 11) and post-operative MRI Brain T1WE axial cut display entire tumor removal.

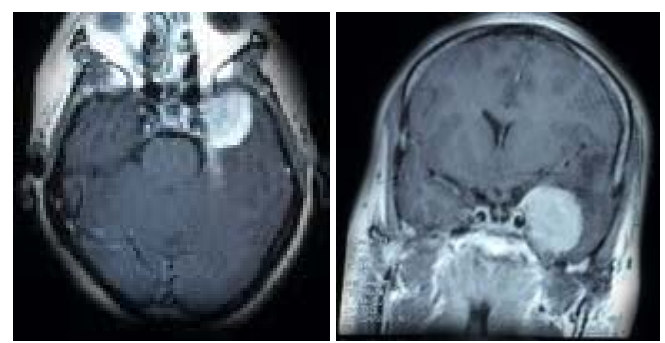

Figure $10-3^{\text {rd }}$ case pre-operative MRI Brain axial \& coronal images display medial sphenoid wing meningioma

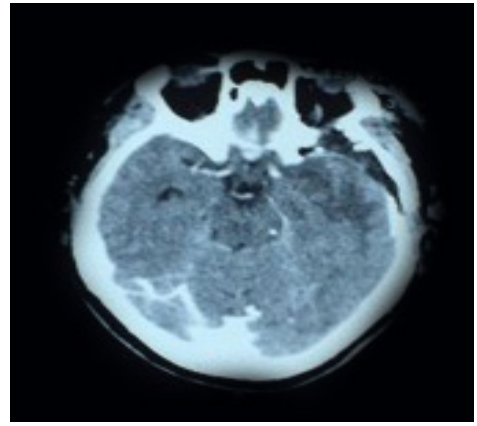

Figure $11-3^{\text {rd }}$ case Post-operative CT Brain axial cut show disappearance of the tumour after the surgery

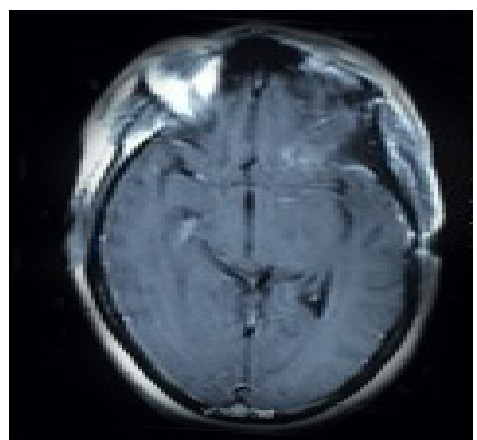

Figure 12 - $3^{\text {rd }}$ case Post-operative MRI Brain axial cut show total tumour excision

\section{Post microsurgical results:}

About the tumours pathological examination in these cases (Table 3) the most common in these cases was Mesothelial meningioma (Figure 13, 14). The second most common was transitional meningioma then fibrous, angiomatous and the least pathology was atypical meningioma that showed aggressive pathological features like more cellular density, partial spiral structure, vascular hyperplasia, necrosis, MIB about $10 \%$ and nuclear enlargement (Figure 15, 16).

\section{TABLE 2}

\section{Histopathological results of the 47 Medial} Sphenoid Wing Meningiomas

\begin{tabular}{|c|c|c|}
\hline Pathology & Number of cases & Percentage \% \\
\hline Mesothelial & 23 cases & $48.9 \%$ \\
\hline Transitional & 12 cases & $25.5 \%$ \\
\hline Fibrous & 7 cases & $14.9 \%$ \\
\hline Angiomatous & 2 cases & $4.25 \%$ \\
\hline Atypical & 3 cases & $6.4 \%$ \\
\hline
\end{tabular}

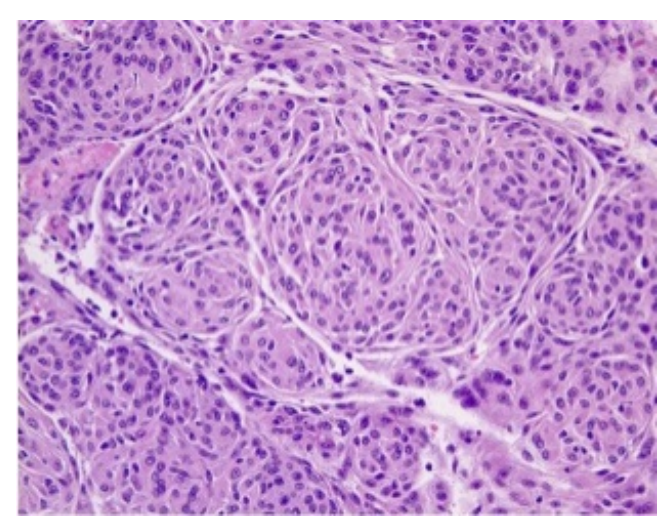

Figure 13 - $100-\mathrm{H} \& \mathrm{E}$ Stain show mild increased cellular density 


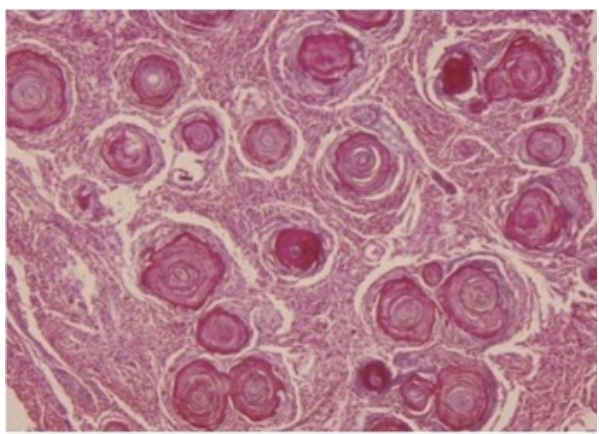

Figure 14 - Characteristic psammoma bodies for meningioma

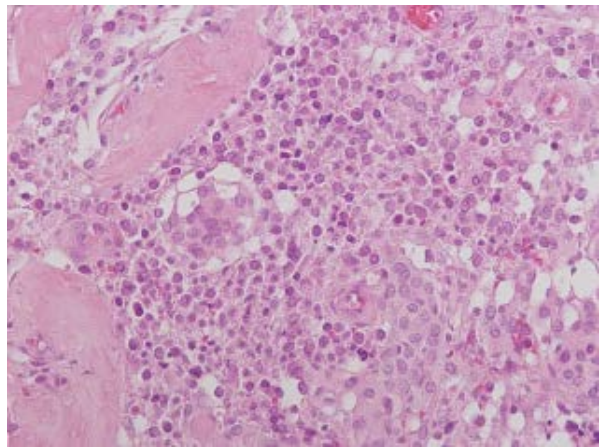

Figure 15 - 200 -H\&E Stain Increase in cell density, Partial spiral structure, vascular hyperplasia, Pattern less pattern, Necrosis, nuclear enlargement. All of the above indicate atypical variant of meningioma

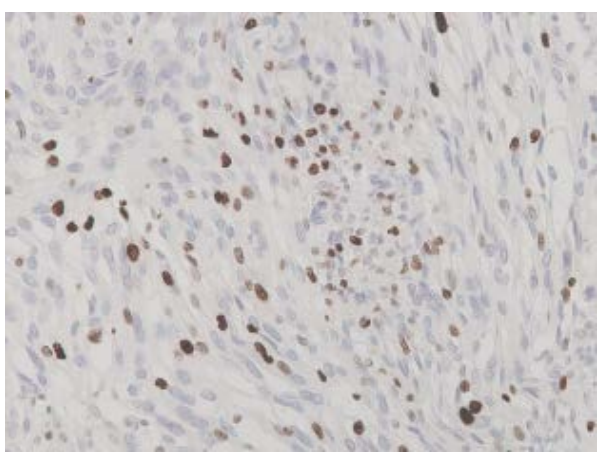

Figure 16 - MIB more than $10 \%$

According to the extent of tumours excision: Total tumor resection (Simpson I-II) was accomplished in 23 cases (48.9\%). On the other hand, (Simpson III) was achieved in 19 cases (40.4\%) and the Partial resection (Simpson IV) was done in 5 cases $(10.6 \%)$. the reason for incomplete removal of tumours in the last two categories may be related to firmness of the tumours, absence of clear arachnoid plane between ICA \& its branches and the tumours leading to adherent of the tumours on the vessels adventitia beside encasement of cranial nerves \& optic apparatus which make complete tumor removal possess a very high morbidity.

Regarding visual acuity post-operative changes: it showed improvement or stable in 27 cases (57.4\%). The neurological deficit remained unchanged postoperative in 35 cases (74.5\%) while improved in 4 cases (8.5\%). The mean Karnofsky Performance Scale (KPS) score for the patients in the study was 86.2 (ranged from 60 to 100) at 1 year after surgery, which was 74.5 at 10 days after surgery.

Our research permanent morbidity represent $17.02 \%$ \& 3 (6.3\%) mortality. All the complication \& its prognosis summarized in (Table 2) and (Figure 17). 13 patients with subtotal resection and 5 patients with partial resection received postoperative radiotherapy.

Tumor recurrent was present in 7 patients (14.9\%). These cases analyzed according to the degree of tumor excision and the results was: a case of complete removal, the 2nd case was incomplete resection and residual 5 cases showed partial removal. The analysis of the same cases in relation to the pathology the results was: 3 cases the pathological investigation was Mesothelial in 3 cases, Transitional in 1 case, Angiomatous in 1 case, Atypical in 2 cases (Table 3). 
TABLE 3

Showed postoperative complications in 47 medial sphenoid wing meningioma cases

\begin{tabular}{|c|c|c|c|}
\hline Complications & $\begin{array}{c}\text { Number } \\
\text { s }\end{array}$ & $\begin{array}{c}\text { Percentages } \\
\mathbf{( \% )}\end{array}$ & $\begin{array}{c}\text { outcomes at } \\
\text { Discharge }\end{array}$ \\
\hline CSF leakage & 3 & $6.4 \%$ & 2 cured \\
\hline Hemiparesis & 5 & 10.6 & $\begin{array}{c}3 \text { improved, } 2 \\
\text { unimproved }\end{array}$ \\
\hline $\begin{array}{c}\text { Incomplete } \\
\text { aphasia }\end{array}$ & 4 & $8.5 \%$ & 2 improved \\
\hline $\begin{array}{c}\text { Cerebral } \\
\text { infarction }\end{array}$ & 2 & $4.25 \%$ & permanent \\
\hline $\begin{array}{c}\text { Epidural } \\
\text { hematoma }\end{array}$ & 3 & $6.4 \%$ & All cured \\
\hline $\begin{array}{c}\text { Tumor bed } \\
\text { bleeding }\end{array}$ & 6 & $12.76 \%$ & All cured \\
\hline
\end{tabular}

\begin{tabular}{|c|c|c|c|}
\hline $\begin{array}{c}\text { Oculomotor } \\
\text { nerve palsy }\end{array}$ & 9 & $19.15 \%$ & $\begin{array}{c}1 \text { cured, } 8 \text { not } \\
\text { improved }\end{array}$ \\
\hline 6th N. palsy & 2 & $4.25 \%$ & 2 cured \\
\hline $\begin{array}{c}\text { Subcutaneous } \\
\text { collection }\end{array}$ & 4 & $8.5 \%$ & 4 cured \\
\hline
\end{tabular}

TABLE 4

Pathology of recurrent cases

\begin{tabular}{|cc|}
\hline pathology & Number of cases \\
\hline Mesothelial & 3 cases \\
\hline Transitional & 1 case \\
\hline Angiomatous & 1 case \\
\hline Atypical & 2 cases \\
\hline
\end{tabular}

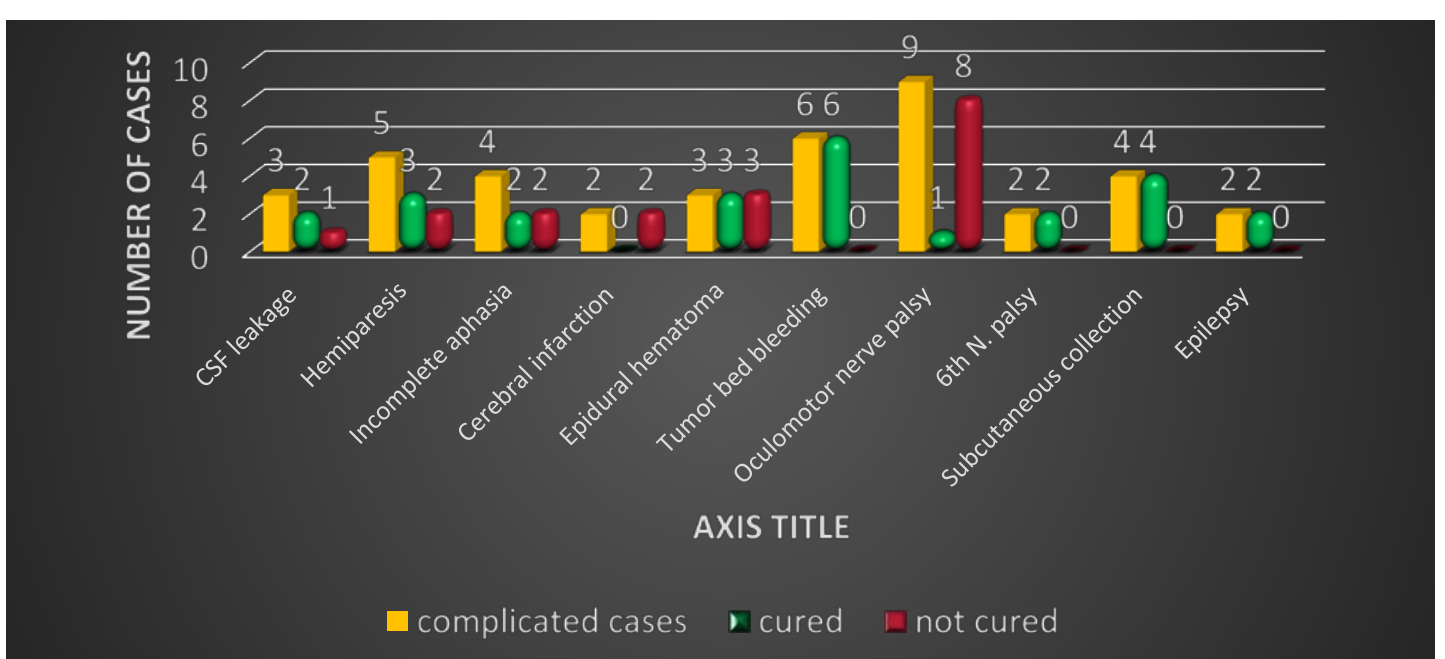

Figure 17 - A diagram display the prognosis of complication in our series

By statistical analysis we document the influence of many factors on postoperative patient's conditions \& survival: Extent of tumor excision $(\mathrm{P}=0.026)$, Peritumoral edema $(\mathrm{P}=0.038$, Vascularity of the tumours $((\mathrm{P}=0.005)$, Size of the tumor $(\mathrm{P}=0.029)$, Presence of arachnoid plane between the tumours and surrounding neurovascular structures $\quad(\mathrm{P}=0.004), \quad$ Preoperative neurological condition $(\mathrm{P}=0.005)$. The factors that did not affect the patient postoperative status include: patients age, sex, duration of symptoms, surgical approach and tumor volume (average $3.7 \mathrm{~cm}^{3}$ ) range between 2.6 $\mathrm{cm}^{3}$ to $6.1 \mathrm{~cm}^{3}$. 


\section{Discussion}

Medial Sphenoid Wing meningiomas (MSWM) had inherited a difficult surgical challenge due to limited critical location that bounded by ON, Clinoid Process, Sphenoid wing, ICA and cranial nerves gone through Superior Orbital Fissure. In spite of its narrow site, it represent over than half of all meningiomas that appear in sphenoid wing. [5]

In the past decades, the total resection of those tumours was not achieved in most of the cases by reason of high morbidity \& mortality related to attempts of radical removal leading the neurosurgeon to prefer partial removal. But the advances in microscopes, microinstruments, modern neurosurgery tools and imaging studies make this goal possible. [4] In our research Total tumor resection (Simpson I-II) was accomplished in 23 cases (48.9\%). Alternatively, (Simpson III) was achieved in 19 cases (40.4\%) and the Partial resection (Simpson IV) was done in 5 cases (10.6\%). The main causes for incomplete excision in our research could be correlated to increased firmness of the tumours, lack of clear arachnoid plane between ICA \& its branches and the tumours leading to adherent of the tumours on the vessels adventitia making total resection impossible without vascular injury that may be fatal beside encasement of cranial nerves \& optic apparatus and inability to remove the intra-cavernous part which make complete tumor removal possess a very high morbidity. One of major factors that affect the extent of tumor removal also was blood loss volume.we made all possible ways to decompress the ON \& all compressed neurovascular structures, maximum intra dural debulking of the tumor (the primary our surgery goals) without burden the patients conditions as we can. These results is comparable to what is mentioned by Brotchi \& Bonnal, Nakamura et al and Sughrue. [14,17] in spite of the old series showed complete removal of the MSWMS fluctuated between 23 to 50\%.[16]. The improvement of the success rate may be related to adoption of microsurgical techniques, invented micro instruments, neuro anesthesia cumulative experience all over the world and in our series may be also to the learning curves of the surgeons. [5, 4]

According to the approach, we did not find any difficulty of meningioma removal because of the selected approach and the approach value in success surgical rate was statistically insignificant. This result opposite to statement by Zhang et al. we can explain that by more homogenous features in our surgical group than Zhang group. We adopt Orbitozygomatic Approach to the tumours that creeping in the middle cranial fossa and extend more posteriorly but the traditional pterional approach was selected to tumours that extend upward. This concept was used to get more detailed view of the ON which can be localized more accurate through the skull base land marks to avoid injury during tumor removal as conducted by the same author. We agree about early devascularization of meningiomas feeders in the skull bas dura was a critical step in reducing blood loss [19] But still extradural anterior clinoidectomy one of the essential steps in all of our approaches in MSWMS 
which approved by Lee et al [10] and against Nakamura et al statement to get more room for dissection and early identification of $\mathrm{ON}$.

The visual impairment represent the commonest preoperative neurological deficit. In literature the paper in last 10 years show further post-operative vision recovery but did not reach the acceptable level.[4,19] In our series, the vision improved or stable in 27 cases (57.4\%). Bassiouni et al reported of 106 cases, VA (Visual Acuity) improved in 21 cases (40.4\%) and Lee et al reported a fair results using a cranial base technique. [3] Our results was obtained thanks for meticulous microsurgical technique, preservation of $\mathrm{ON}$ nutrient feeders and avoidance of cautery whenever possible. $\mathrm{Li}$ et al gave same explanation in his series. [12]

The neurological deficit in our cases remained permanent postoperatively in 35 cases (74.5\%) while improved in 4 cases (8.5\%). Our research morbidity represent $17.02 \%$ \& 3 cases (6.3\%) mortality. Al-Mefty et al stated morbidity rate of $25 \%$ and mortality rate $42.9 \%$ in a his series of 24 cases and testified that "while Czernicki et al reported a low mortality rate which was $6.7 \%$. The complications after surgery were related with the extent of tumor resection". [2] While other international authorities conducted a mortality rate that reach $15.4 \%$ and the frequency of permanent profound neurological deficit fluctuating between $8.8 \%$ and $60 \%$. [8] Our data was comparable to these results. The mortality and morbidity had a very close relation to the cavernous sinus tumor portion beside in ability to dissection of neurosurgical bundles from the tumor, spasm of perforators or /and vascular injury. We minimize this issue by adequate drilling of the sphenoid bone, Anterior clinoid removal to get space and early identification of neural \& vascular structures to decrease its injury during tumor removal that most of the time may mask its location.

The most common complication in our cases was 3 rd N. Palsy that was in 4 cases (8.5\%) preoperative and became 9 (6 total -3 partial) cases (19.15\%) immediate postoperative then improved ( 1 cured -3 total -5 partial ). these results may be higher than some paper of the same interest as Zhang et al who stated post-operative $3 \mathrm{rd} \mathrm{N}$. Palsy in $18.9 \%$ but improve in most of the cases and reduced to 4 cases only suffered from 3rd N. Palsy[8].this may be as a result thermal injury during drilling the lip of Superior Orbital Fissure (SOF) and anterior clinoidectomy, injury of 3rd $\mathrm{N}$. in the meningioma cavernous part $[1,3,7,14,18]$ or/and surgical manipulation or cautery at the tent edge where $3 \mathrm{rd} \mathrm{N}$. pass to enter the oculomotor triangle and SOF. So it wise to leave adherent cavernous sinus tumor part to decrease postoperative $3 \mathrm{rd} \mathrm{N}$. impairment.

CSF leak recorded in 3 cases in our research which appeared in many extensive skull base approaches. We treat it with lumbar drain for few days then Acetazolamide tablets 500mg$1000 \mathrm{gm}$ according to body weight as oral tablets /per day under umbrella of broad spectrum antibiotic. Two of the three cured while the 3rd case developed meningitis and we EVD (External Ventricular Drainage) device and the patient died. Nakamura et al mentioned a case of post-operative CSF Leak 
who treated by endoscopic endonasal transsphenoid repair [14] and Sughrue et al also a case treated conservatively. May be the difference because we did not use fibrin glue in early cases to seal the skull base dura and also we adopt lumbar drain to decrease the incidence of CSF Leak.

6th N. Palsy occurred in 2 patients postoperatively. thus may be related to brain edema postoperative and both patient treated conservatively till the edema subside then the 6th N improved spontaneously as documented by Liu $\mathrm{D}$ et al who gave the same report.

5 cases suffered from hemiparesis as postoperative condition 3 of them had weakness preoperative after few weeks 3 cases improved. Incomplete aphasia occurred in 4 cases (8.5\%) 2of them improved We assumed the surgical manipulation around perforator in territory of MCA (Middle Cerebral Artery) may trigger spasm in some extent, peritumoral edema was in optimum level postoperative that burden the patients neurological conditions beside the effect of retractors. during the series upgrade we adopt more skull base bone removal to minimize brain retraction, minimize coagulation to reduce the incidence of new neurological deficit. Many paper mentioned these complications in similar surgeries for MSWM as Nakamura et al, Czernicki et al, Bassiouni et al, Hao, Liu et al. $[3,7,8,11,14]$ may be it appeared in ours and in Nakamura et al study due to presence of larger tumours that extend posteriorly and superiorly wit adherence to MCA or ACA perforators or to the MCA or ACA adventitia [2]. Thus also explain the presence of two cases of cerebral infarction which was evident clinically \& radiologically. They died few weeks after the surgery and the case of death in patient was massive cerebral edema with massive infarction \& the other case was sever chest infection in 67 years old male with history of heavy smokers and Bronchial Asthma. Liu et al conducted 6 patient died due to recurrence or progression of the tumours and 2 died of unrelated diseases [11]

There was 3 case of acute epidural haematoma: two case treated surgically and a case managed conservatively. The bleeder was the stem of Middle meningeal in two cases due to retraction in foramen spinosum. In 3rd case, the bone sinuses was the source of bleeding. The tumor bed haemorrhage occurred in 6 cases. 5 cases managed conservatively because of there was no midline shift or progressive increasing of the volume may be due to general oozing without identified the source of bleeding while one case treated surgically due to progressive enlargement of the haematoma and the source was small perforator. Nakamura testified 2 cases of mortality: a case of MCA stroke \& haemorrhage in tumor bed and the other because of cerebral edema on the other hand Kim et al reported 5 cases of postoperative haemorrhage/infarction (8.5\%) without details about the cause or the criteria [9]. The cause of intracerebral tumor bed haematoma the well of the surgeon to minimize and meticulous bleeding control to avoid postoperative strokes and worsening of the patients' neurological condition. Subcutaneous collection appeared in 4 cases (8.5\%) three of them treated conservatively and only 1 managed by surgical wound 
revision. Sughrue et al reported 4 cases of wound infection and a case of wound dehiscence. [17]

We did not have any cases suffered from postsurgical intracranial hypotension, hyponatremia or the syndrome of inappropriate antidiuretic hormone secretion (SIADH) as reported by Czernicki et al and this might be due to small number of our cases. [7]

Histopathological examination showed the most common type was WHO Grade I Mesothelial meningioma in 23 cases (48.9\%) as reported by many authorities. $[11,14,17]$ but we had three cases of atypical meningioma which different from Nakamura et al who did not report any cases of atypical meningioma [14]

In patients long-term prognosis regarding the quality of life: Over all the mean KPS score for the patients in the study was 86.2 (ranged from 60 to 100) at 1 year after surgery, which was 74.5 at 10 days after surgery. While AbdelAziz et al stated excellent outcomes 38 patients, with a KPS score $>90$ in $89 \%$ of their cases 1 year after surgery [1]and Liu et al showed In 120 MSWMS cases mean KPS was 83.5 at 10 days after surgery then improved to be 90.6 as mean KPS (ranged from 60 to 100) 12 months after [11].so our results may goes with international data because of advanced microsurgical techniques, learning curve with preservation of perforators whenever possible and appropriate decision making according to patient condition.

13 patients subtotal resection and 5 patients with partial resection received postoperative radiotherapy as we applied benefit of radiotherapy on enhancing the patients survival as approved by Pamir et al in his series [15] but we did not notice the radiotherapy problem in follow up and we believed the detailed results of radiotherapy may be is not our research focus.

7 patients (14.9\%) showed tumor recurrence. This recurrence have a close relation to the extent of tumor excision (5 cases with previous partial removal, a case of former complete removal, last case had earlier incomplete resection). Also it related to involvement of cavernous sinus, grade of meningioma (2 cases with Atypical meningioma had recurrence). In the case of complete removal who showed recurrence the tumorous hyperostotic bone \& dura may be the nidus for regrowth. Nakamura et al reported the high rate of recurrence $(27.5 \%)$ of clinoidal meningioma related to involvement of cavernous sinus as only cause in comparison to low rate of tumor recurrent in case showed no cavernous sinus involvement (7.7\%) [2] While Liu et al mentioned the close relation between recurrence in his series and tumor Atypia [11]

By analyzing the data statistically, we found the influence of several factors on postoperative patient's prognosis: Extent of tumor excision $(\mathrm{P}=0.026)$ and we can explained that by the major effect of residual volume to promote the chance of recurrence as mentioned earlier, Peritumoral edema $(\mathrm{P}=0.038)$ due to Lower blood flow to the brain, Limited space for retraction \& Higher incidence of brain contusion during surgery (we can overcome this by more removing skull base bone as total drilling of sphenoid wing, 
clinoidectomy). The Vascularity of the tumours represent one of the main factors affecting our patients prognosis $(\mathrm{P}=0.005)$ especially internal carotid parasitic blood supply that may be difficult to identify \& control intraoperative beside its sometimes related to perforators. The Size of the tumor $(\mathrm{P}=0.029)$ because of major neurovascular involvement, cavernous sinus invasion \& Rich blood supply internal \& external carotid that lead to more blood loss and sometimes limit the surgical excision as conducted by Pamiret al\& Goel et al [15]. The Presence of arachnoid plane between the tumours and surrounding neurovascular structures $(\mathrm{P}=0.004)$ play also important role in the series results due to its facilitator effect in tumor total excision, reduce the vasospasm by isolating the neurovascular bundles that limiting the surgical manipulation in these vital structures with subsequent reduction of vasospasm \& Cranial nerves injury. The Preoperative neurological condition was $(\mathrm{P}=0.005)$ as it was a reflection of invasion \& involvement of neurovascular structure like optic nerve ON, other cranial nerves and cranial vascular tree. The factors that did not affect the patient postoperative status include: patients age, sex, duration of symptoms, surgical approach. Many researchers conducted the importance of some of these factor on patient survival and quality of life like Sughrue et al Nakamura et al, Zhang etal, Liu D et al $[9,13,11,17,19]$ but our result disagree with Kim et al about age as influencing factor [9]

Conclusion: There are many factors influence the prognosis of inner sphenoid wing meningioma including the extent of surgical resection, cavernous sinus invasion, present or absent of peritumoral edema, vascularity of the tumor, size of the tumor, presence or absence of arachnoid plane between the tumor and surrounding neurovascular structures and preoperative neurological condition.

\section{Correspondence}

Ashraf ElBadry, M.D., IFAANS, Osaka University fellow

Address: Department of neurological surgery,

Faculty of medicine, Mansoura university hospital, EGYPT

Email: ashrafbadry@hotmail.com;

ashrafbadry@mans.edu.eg

Cell phone: $+201111300033-+201223477444$

\section{References}

1.Abdel-Aziz KM, Froelich SC, Dagnew E, Jean W, Breneman JC, Zuccarello M, et al. Large sphenoid wing meningiomas involving the cavernous sinus: conservative surgical strategies for better functional outcomes. Neurosurgery 2004; 54(6):1375-83.

2.Al-Mefty O, Smith RR: Surgery of tumors invading the cavernous sinus. Surg Neurol 30:370-381, 1988

3.Bassiouni H, Asgari S, Sandalcioglu IE, Seifert V, Stolke D, Marquardt G: Anterior clinoidal meningiomas: Functional outcome after microsurgical resection in a consecutive series of 106 patients. Clinical article. J Neurosurg 111:1078-1090, 2009

4.Behari S, Giri PJ, Shukla D, Jain VK, Banerji D: Surgical strategies for giant medial sphenoid wing meningiomas: A new scoring system for predicting extent of resection. Acta Neurochir (Wien) 150:865-877, discussion 877, 2008

5.Benjamin V, McCormack B: Surgical management of tuberculum sellae and sphenoid ridge meningiomas, in Schmidek HH (ed): Operative Neurosurgical Techniques: Indications, Methods, and Results. Philadelphia, W.B. Saunders, 2000, pp 305-315.

6.Crooks, V, Waller S, Smith T, Hahn TJ: The use of the Karnofsky Performance Scale in determining outcomes 
and risk in geriatric outpatients. J Gerontol 46: M139-144, 1991

7.Czernicki T, Kunert P, Nowak A, Marchel A. Results of surgical treatment of anterior clinoidal meningiomas our experiences. Neurol Neurochir Pol.2015; 49(1):29-35. Doi: 10.1016/j.pjnns. 2015.01.003. Epub 2015 Jan 15. Pub Med PMID: 25666770.

8.Hao S, Tian R, Wu Z, et al. Clinical characteristics and prognosis factors analysis for post-operative ptosis of sphenocavernous meningiomas: a single institution study. Clin Neurol Neurosurg 2015; 131:35-41.

9.Kim JH, Jang WY, Jung TY, Kim IY, Lee KH, Kang WD, Kim SK, Moon KS, Jung S.Predictive factors for surgical outcome in anterior clinoidal meningiomas: Analysis of 59 consecutive surgically treated cases. Medicine (Baltimore). 2017Apr; 96(15):e6594. Doi: 10.1097/ MD.00000000000065 -94. PubMed PMID: 28403097; PubMed Central PMCID: PMC5403094.

10.Lee JH, Jeun SS, Evans J, Kosmorsky G: Surgical management of clinoidal meningiomas. Neurosurgery 48:1012-1019, 2001.

11.Liu DY, Yuan XR, Liu Q, Jiang XJ, Jiang WX, Peng ZF, Ding XP, Luo DW, Yuan J. Large medial sphenoid wing meningiomas: long-term outcome and correlation with tumor size after microsurgical treatment in 127 consecutive cases. Turk. Neurosurg. 2012; 22(5):547-57. Doi: 10.5137/1019-5149.JTN.5142-11.1. PubMed PMID: 23015330.

12.Li X, Liu M, Liu Y, Zhu S: Surgical management of tuberculum sellae meningiomas. J Clin Neurosci 14(12):1150-1154, 2007
13.Malik I, Rowe JG, Walton L, Radatz MWR, Kemeny AA: The use of stereotactic radiosurgery in the management of meningiomas. Br J Neurosurg 19(1):1320, 2005

14.Nakamura M, Roser F, Jacobs C, Vorkapic P, Samii M: Medial sphenoid wing meningiomas: clinical outcome and recurrence rate. Neurosurgery 58:626-639, 2006 15.Pamir MN, Belirgen M, Ozduman K, Kiliç T, Ozek M. Anterior clinoidal meningiomas: analysis of 43 consecutive surgically treated cases. Acta Neurochir 2008;150(7):625-35.

16.Pompili A, Derome PJ, Visot A, Guiot G: Hyperostosing meningiomas of the sphenoid ridge: Clinical features, surgical therapy, and long-term observa- tions: Review of 49 cases. Surg Neurol 17:411416, 1982.

17.Sughrue ME, Rutkowski MJ, Chen CJ, Shangari G, Kane AJ, Parsa AT, Berger MS, McDermott MW. Modern surgical outcomes following surgery for sphenoid wing meningiomas. J Neurosurg. 2013 Jul; 119(1):86-93. Doi: 10.3171/2012.12.JNS11539.Epub 2013 Feb 22. PubMed PMID: 23432379.

18.Tobias S, Kim CH, Kosmorsky G, et al. Management of surgical clinoidal meningiomas. Neurosurg Focus 2003; 14:e5.

19.Zhang J, Shrestha R, Cai BW, Zhou PZ, Li YP, Jiang S. Management of large medial sphenoid wing meningiomas: a series of 178 cases. Turk Neurosurg. 2014; 24(5):664-71. Doi: 10.5137/1019-5149.JTN.766813.2. PubMed PMID: 25269034. 